\title{
THE MAGNETIC FIELD OF THE SUN: AN OBJECT LESSON
}

\author{
E.N. Parker \\ Laboratory for Astrophysics \\ University of Chicago \\ 933 East 56th Street, Chicago, Illinois 60637, USA
}

\begin{abstract}
The magnetic field of the sun is created by a magnetohydrodynamic dynamo under conditions bearing some qualitative similarities to the apparent generation of the galactic field in the gaseous disk of the galaxy. There is a similarity, too, in the extension of bipolar lobes of the solar field above the surface of the sun and the extension of bipolar lobes of the galactic field outward from both sides of the disk. Hence one can learn a lot about the expected origin and activity of the galactic field by studying the behavior of the magnetic field of the sun. In particular, the mysteries associated with the "simple" circumstances of the origin of the solar magnetic field far below the surface are no less than the mysteries in the theoretical origin of the galactic field, where there is so little direct observation of the small scale motions and magnetic fields. There is reason to think that the activity of the magnetic field of the sun, producing prominences, flares and X-ray corona, a solar wind, and coronal mass ejection may all have counter parts in the activity of the galactic field above the surface of the gaseous disk.
\end{abstract}

A review of the magnetic fields of the sun at an IAU Symposium on galactic and intergalactic fields has two obvious purposes. One is to demonstrate the complexity and mystery of magnetic behavior in a convecting rotating body like the sun, which suggest the comparable complexity and mystery of the magnetic behavior of the galaxy. Second, the similarity of some of the general conditions in the sun and in the galaxy suggests the specific character of some aspects of the magnetic activity of a galaxy. The scale of galactic fields is, of course, different from the scale of the magnetic fields of the sun. However the comparison is based not on the scales but on the large magnitude of the magnetic Reynolds number in both cases and on the rough equality of the turbulent pressure and magnetic pressure throughout the dynamo region. In particular, the effective magnetic Reynolds number (taking account of turbulent diffusion and neutral point reconnection) is essentially the same in both cases. The primary magnetohydrodynamic differences between the sun and the galaxy are the long time scale of the galactic activity, so that observations 
provide only a single snapshot, and the powerful flow of radiation through the enormous optical depth of the sun in contrast with the transparency of the galaxy.

To begin, it is believed that the magnetic field of the sun is produced by a magnetohydrodynamic dynarno, combining cyclonic convection (the $\alpha$-effect) with nonuniform rotation (the $\omega$-effect) (Parker, 1955, 1957a, 1979a; Moffatt, 1978; Krause and Rädler, 1980). A similar scheme is believed to be the cause of the present magnetic field of the galaxy (Parker, 1971a,b, 1979a; Stix, 1975; White, 1978; Soward, 1978; Ruzmaikin, Shukurov, and Sokoloff, 1988). It should be noted, then, that recent advances in precision observations of the oscillations at the visible surface of the sun raise questions concerning the nature and location of the solar dynamo. To be precise, helioseismology shows only a slow decrease of angular velocity $\Omega$ downward through the convective zone (Duvall, et al. 1984), in contrast to the previously conjectured downward increase. The slow downward decrease of $\Omega$ combined with the converging flow at the base of a rising convective column provides a $2 \mathrm{~m} / \mathrm{sec}$ equatorward migration of the bands of azimuthal field (just as a downward increase of angular velocity combined with the expansion of a rising column of fluid provides equatorward migration) in accord with the equatorward migration of the two bands of magnetic activity oiserved at the surface of the sun. On the other hand, the phase of the poloidal fields, deduced from such a dynamo model does not agree entirely with the observed phase of the polar fields of the sun. What is more, the recent work of Choudhuri and Gilman (1987) raises questions as to whether the surface activity on the sun tracks the magnetic field at depth. Their calculations indicate that a rising loop of field is driven by the Coriolis force to move along a path almost parallel to the axis of rotation. Another perplexing point is the unknown meridional circulation in the sun, which numerical simulations indicate may be in either direction (toward or away from the equator depending upon the model) at magnitudes of $5 \mathrm{~m} / \mathrm{sec}$ or more (Gilman, 1983; Glatzmaier, 1985). Such circulation dominates the $2 \mathrm{~m} / \mathrm{sec}$ equatorward migration of the bands of magnetic activity. The dynamo may have little or nothing to do with the observed equatorward 2 $\mathrm{m} / \mathrm{sec}$ (Parker, 1987a).

Another problem arises from the large quantity of magnetic flux that is observed to well up through the surface of the sun in a large, long-lived activity complex (bipolar magnetic region). Gaizauskas, Harvey, Harvey and Zwaan (1983) provide quantitative observations of such an active region, showing intermittent eruptions of $10^{22}$ Maxwells at irregular intervals of $10^{6} \mathrm{sec}$ and a total magnetic flux of $8 \times 10^{22}$ Maxwells through the surface at one time. This quantity of magnetic flux appearing at the surface implies an azimuthal flux not less than about $2 \times 10^{23}$ Maxwells beneath the surface. Needless to say, there is no unique upper limit on the azimuthal flux that may be hidden beneath the surface of the sun. The essential point is that $2 \times 10^{23}$ Maxwells is already a large magnetic flux. If it is drawn from a latitudinal 
band of $5^{\circ}$ width (about $5 \times 10^{4} \mathrm{~km}$ ) and a radial depth of $10^{5} \mathrm{~km}$ (e.g. the lower half of the convective zone), the azimuthal field strength is at least $3 \times 10^{3}$ gauss (Parker, 1987b). This is a strong magnetic field, about equal to the equipartition value at the maximum of $\frac{1}{2} \rho v^{2}$ in the middle of the convective zone (cf. Spruit, 1974). It is apparent that so strong an azimuthal field inhibits the convection and the associated convected heat transport. The result is a cool shadow above the band of azimuthal field, the weight of which is sufficient to suppress the buoyancy of an azimuthal field up to about $5 \times 10^{3}$ gauss (Parker, 1987b,c). The accumulation of heat underneath is not so strong as the cool shadow, so the downward force of the shadow prevails. But the accumulation of heat does produce a Rayleigh-Taylor instability of the lower side of the azimuthal field, causing eruption of loops of field to the surface at intervals of the order of $10^{6} \mathrm{sec}$ (Parker, 1987b,d). This is evidently the basis for the observed intermittent emergence of magnetic flux at the surface at irregular intervals of $10^{6} \mathrm{sec}$ to maintain the long lived activity complexes (e.g. the region studied by Gaizauskas, et al, 1983). Note, then, that the bipolar loops of magnetic field at the surface of the sun, which are the principal architects of solar activity, are a consequence of the special conditions to be found in the convective zone of the sun.

The large azimuthal flux ( $>10^{23}$ Maxwells) in each hemisphere makes the theoretical solar dynamo problem particularly puzzling. There is no formal hydrodynamic theory for the turbulent convection in the convective zone of the sun. The present models of the convection are based on little more than dimensional analysis. It is doubly difficult, therefore, to understand the turbulent diffusion of the strong solar magnetic field and to understand the quantitative $\alpha$-effect in the same strong field. Both are essential parts of the solar dynamo. So contemporary dynamo theory is based on dimensional analysis, for both the dynamo coefficient $\alpha$ and the turbulent resistive diffusion coefficient $\eta$. It is entirely possible that the current concepts are quantitatively incorrect, and indeed there may be some qualitative misconceptions as well. The difficulty is compounded by the considerable strength of the field with which the convection is obliged to grapple.

The same problem arises in the gaseous disk of the galaxy where the azimuthal field is comparable to the equipartition value $\left(4 \pi \rho v^{2}\right)^{\frac{1}{2}}$ of the disordered interstellar motions, and is therefore resistant to deformation by the turbulence. Again, both the turbulent diffusion $\eta$ and the $\alpha$-effect are essential for generation of the galactic field. So the theoretical origin of the galactic field contains much the same uncertainties as the origin of the field of the sun.

The magnetic field of the sun is an intense fibril state where it passes through the surface. The individual fibrils of $1-2 \times 10^{4}$ gauss (Beckers and Schröter, 1968; Stenflo, 1973) have diameters of the general order of $2 \times 10^{2} \mathrm{~km}$, well below the limit of resolution of the observing magnetometer. The observed mean field is 
then a measure of the relative spacing of the unresolved fibrils. The concentration of magnetic field into the fibril state represents an enhancement of the magnetic energy by factors of 10 to $10^{2}$, for a given mean field of $10-10^{2}$ gauss respectively. On the other hand, the fibril state inhibits the convective heat transport less than the same total flux in continuous form. Simple estimates indicate the observed fibril state represents a minimum of the total energy (Parker, 1984). The effect seems to be forced on the field by the heat transport at the radiative surface of the sun (Spruit, 1979; Deinzer et al 1984a,b; Hasan, 1985). There is no compelling reason to think that the field is an intense fibril state throughout the convective zone. Freshly emerging magnetic flux shows intensities of only $5 \times 10^{2}$ gauss (Brants, 1985; Zwaan, 1985). There is no reason whatever to think that the galactic field is forced into a fibril state in the interstellar medium.

Sunspots, whose existence has been known for about two thousand years, are formed by the spontaneous (and entirely puzzling) clustering of individual fibrils (Zwaan, 1978, 1985). The clustering occurs only while fresh flux is emerging in the region. With the cessation of flux emergence the clustering reverses and the sunspot comes apart on time scales as short as hours or as long (in the case of large circular spots) as weeks (Vrabec, 1971; Harvey and Harvey, 1973; Zwaan, 1978). The only suggestion for understanding the clustering is a powerful and unseen converging convective flow at a distance of the order of $10^{4} \mathrm{~km}$ below the surface (Meyer et al, 1974; Parker, 1979b). There is no analogous galactic effect to be expected.

The most significant similarity between the sun and the galaxy is in the general qualitative nature of the magnetic activity above the surface. The surface of the sun is densely speckled with bipolar fields to $10^{2}$ gauss on all scales from the $2 \times 10^{5} \mathrm{~km}$ of the large "normal" active region down to the smallest scales of $2 \times 10^{8} \mathrm{~km}$ resolved by the magnetometer (fluxes of $6 \times 10^{22}$ Maxwells down to $10^{18}$ Maxwells or less). The large bipolar magnetic regions appear in isolation at longitude intervals of the order of $45^{\circ}$ (Gaizauskas, et al 1983), but the small bipolar regions interact with each other, frequently changing their magnetic topology through reconnection between temporary neighbors. The individual small bipoles appear and disappear on time scales of several hours, presumably emerging through the surface and subsequently sinking below the surface again, perhaps with different magnetic connections from when they emerged. Bipoles with dimensions of several thousand $\mathrm{km}$ often contain $\mathrm{X}$-ray emitting gases $\left(10^{6}{ }^{\circ} \mathrm{K}\right)$ near their apexes. The larger stronger bipoles always contain X-ray emitting gas, with the emission depending on the strength of the magnetic field (Rosner, Tucker, and Vaiana, 1978). A curious feature of the X-ray brightness is that it is about the same for all bipolar fields with dimensions between $6 \times 10^{3} \mathrm{~km}$ and $2 \times 10^{5} \mathrm{~km}$.

As already noted, the large bipolar active regions on the sun are often maintained for many months by repeated eruptions of bipolar fields (presumably from the az- 


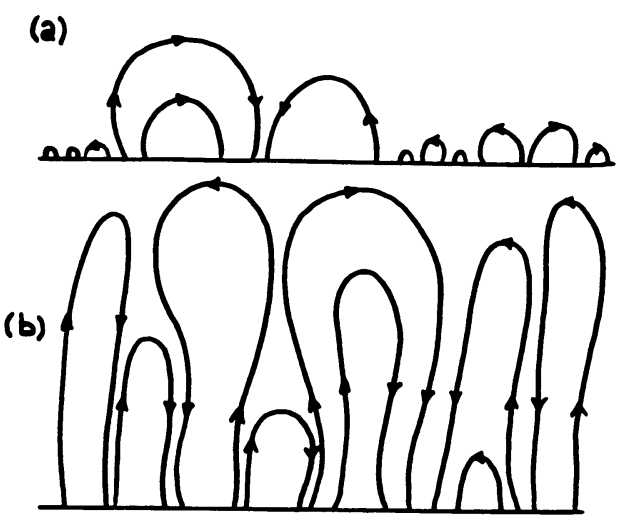

Fig. 1: (a) A schematic of the bipolar magnetic fields extending above the surface of the sun and (b) from the gaseous disk of the galaxy.

imuthal field in the lower convective zone) at intervals of the order of $10^{6} \mathrm{sec}$, as a result of a complicated thermal relaxation oscillation.

The situation in the gaseous disk of the galaxy is different, but with much the same final result. The surface of the gaseous disk of the galaxy is expected to be densely covered by bipolar loops of field, with the loops extending upward through the surface of the disk between regions of dense interstellar gas, at intervals of $0.5-1.5 \mathrm{kpc}$. This general structure is largely a result of the dynamical instability of a gravitationally confined horizontal magnetic field (Parker, 1966, 1968, 1969). The outstanding feature is the rapid inflation of the bipolar loops by the cosmic rays generated in the disk (presumably by supernovae, etc.) to produce an extended galactic halo (Parker, 1965, 1968). The essential point is that the observed breakage of the heavier nuclei among the cosmic rays indicates that the cosmic rays have passed through about $5-6 \mathrm{gm} / \mathrm{cm}^{2}$ of matter, presumably interstellar matter. A mean gas density of two hydrogen atoms $/ \mathrm{cm}^{3}$ yields a dwell time $t$ of about $2 \times 10^{6}$ years for a cosmic ray particle moving with a speed comparable to $c$. It follows that the cosmic ray gas occupying the disk must be replaced in a time $t$. Cosmic rays can escape only by pushing their way out of the disk, of half thickness $h \cong 10^{2}$ pc. This they do by inflating the outward bulging bipolar loops of field at a rate $h / t \cong 50 \mathrm{~km} / \mathrm{sec}$. Eventually the extended loops cut loose from the disk by magnetic reconnection, but that is a slow process so they extend far out from the disk (many kps). This is the origin of the halo of the galaxy. Fig. 1(a) presents a sketch of the general nature of the bipolar fields on the sun and Fig. 1(b) sketches the form of the bipolar loops that make up the halo of the galaxy, for purposes of comparison and contrast. 
In the sun the bipolar magnetic fields extending above the surface are sheared and distorted by the random continuous convective transport of their footpoints in the photospheric convection and in the nonuniform rotation of the sun. In the galaxy the extended bipolar magnetic fields are sheared and distorted by the motions of their footpoints in the randomly moving interstellar clouds and in the nonuniform rotation of the galaxy. In addition they are inflated nonuniformly by the cosmic rays.

The thermal instabilities (Parker, 1953; Field, 1965) of the chromospheric and coronal gases of the sun produce concentrations of cool luminous gas which can be seen streaming downward toward the sun unless supported by a suitable magnetic configuration, in which case it forms a prominence. Sufficient shearing and twisting of a bipolar field can produce a coronal mass ejection (see paper by B.C. Low, these Proceedings). When two bipoles with the same sign press together, there is a current sheet (tangential discontinuity) formed between them which may produce a flare, with intense heating ( $6010^{7}-10^{8} \mathrm{~K}$ ) and acceleration of particles, occasionally to relativistic energies (Parker, 1957b; Sweet, 1958, Dungey, 1958). We may expect similar effects to arise in the halo of the galaxy, as a consequence of the same general deformed condition of the magnetic fields.

In particular, we wish to call attention to the spontaneous appearance of tangential discontinuities as an intrinsic part of the static equilibrium of a magnetic field (embedded in a highly conducting fluid) that is subject to any but the most carefully tailored deformation (by inflation and/or motion of the footpoints) (Parker, 1972, 1979a, 1982, 1983a,b, 1989, 1990). The unavoidability of tangential discontinuities can be seen from the equation $\nabla \times B=\alpha B$ for a force-free field, where $\alpha$ is the torsion coefficient $\alpha=\mathbf{B} \cdot \nabla \times \mathbf{B} / B^{2}$. The divergence of this equation yields the well known result $(\mathbf{B} \cdot \nabla \alpha=0)$ that $\alpha$ is constant along each line of force. But in any but the simplest deformations of a field, there are lines of force wrapping first one way and then the other around neighboring flux bundles. The torsion coefficient cannot accommodate both signs and the field avoids the difficulty by forming a tangential discontinuity, i.e. a current sheet, in which $\alpha$ has the form of a delta function. The tangential discontinuity does not violate $\mathbf{B} \cdot \nabla \alpha=0$ because it contains no magnetic flux. As a matter of fact, tangential discontinuities appear in even simpler situations (see refs. in Parker, 1990). The essential point is that the force-free bipolar magnetic fields, in which the active X-ray corona of the sun is formed, must be full of small tangential discontinuities as a consequence of the continual motion of their footpoints. Evidently it is the dissipation at these discontinuities through neutral point reconnection etc. that is the primary heat source responsible for creating the $\mathrm{X}$-ray corona $\left(10^{10}\right.$ atoms $/ \mathrm{cm}^{3}$ at $2-3 \times 10^{6}{ }^{\circ} \mathrm{K}$ in fields of $10^{2}$ gauss) (Glencross, 1975; Parker, 1981, 1983a). 
The traditional explanation for coronal heating has been the dissipation of waves, propagating upward from the convective zone. However, only the Alfvén waves are expected to make it as far as the corona, and the problem is that they are not inclined to dissipate once they arrive. The observational fact is that bipolar fields on all scales from the arge normal active region at $2 \times 10^{5} \mathrm{~km}$ down to the small ephemeral active region, or X-ray bright point, at $6 \times 10^{3} \mathrm{~km}$, have about the same $\mathrm{X}$-ray brightness, of $10^{7} \mathrm{ergs} / \mathrm{cm}^{2}$ sec (Rosner, Tucker and Vaiana, 1978). The period of a photospheric granule, presumably responsible for generating the waves, is of the order of $300 \mathrm{sec}$. Oscillations with periods as short as $50 \mathrm{sec}$ are detected, but nothing with a shorter period has been found so far. The Alfvén speed in a bipolar magnetic region is of the order of $2 \times 10^{3} \mathrm{~km} / \mathrm{sec}$, so the shortest wavelength (for a period of $50 \mathrm{sec}$ ) is about $10^{5} \mathrm{~km}$. Such long waves cannot heat the smaller bipoles. Yet bipoles are about equally bright on all dimensions. It appears, then, that the $\mathrm{X}$-ray corona is not primarily a wave phenomenon, and we must look elsewhere for the principal source of heat.

The tangential discontinuities appear to be the main heat source. On this basis, the solar X-ray corona is to be understood as the result of a large number of small impulsive reconnection events of $10^{23}-10^{25}$ ergs per event. We have referred to the individual burst of heat as a nanoflare (recalling that the term microflare is applied to flare events at $10^{27} \mathrm{ergs}$ ). The $\mathrm{X}$-ray corona is, then, a cloud of nanoflares (Parker, 1988), and ojservations at high space and time resolution indicate the small scale impulsive nature of coronal heating. Indeed, the same impulsive heating evidently appears in a flare. Machado et al $(1988 \mathrm{a}, \mathrm{b})$ point out that while the most intense flare emission is from the main current sheet between interacting bipoles, the main total energy release of a flare is softer and more diffuse, filling one or more of the interacting bipoles. We have suggested (Parker, 1987e) that this part of the flare is the result of the occurrence of simultaneous nanoflares throughout the bipole, ignited by the general deformation of the bipole and perhaps by the agitation produced at the central current sheet.

Similar tangential discontinuities are expected in the extended bipolar magnetic fields that make up the halo of the galaxy. The principal energy input is the outward expanding cosmic ray gas, estimated to be produced at a rate of the order of $3 \times 10^{40} \mathrm{ergs} / \mathrm{sec}$ (in order to replenish the volume of the gaseous disk to a density of $2 \times 10^{-12} \mathrm{ergs} / \mathrm{cm}^{3}$ in a time of $2 \times 10^{6}$ years). It follows that the heating and the X-ray emission from the halo of the galaxy should be of the same order. And like the X-ray corona of the sun, the X-ray corona of the galaxy is made up of a swarm of galactic "nanoflares."

In summary, there are basic qualitative similarities between the creation of the solar and galactic magnetic fields by a magnetohydrodynamic dynamo process between the general occurrence of bipolar magnetic fields over the surface of the sun 
and the disk of the galaxy, and between the general activities of the surface bipolar magnetic fields. Prominences flares, microflares, and nanoflares are a feature of the sun, and their galactic counterparts may be expected in the galactic halo.

The corona of the sun produces a solar wind and one expects that the galaxy has a similar outflow (see the paper by H.J. Völk, these proceedings).

Needless to say, there are profound differences between the activity of the sun and the activity of the galaxy, as noted in the introduction. But it is worthwhile keeping the possible similarities in mind as the observations of galactic activity progress in the coming years. It should be a useful scientific aid in interpreting the observations and in planning observational programs.

\section{REFERENCES}

Beckers, J.M. and Schröter, E.H. (1968), Solar Phys. 4, 142-167.

Brants, J.J. (1985), Solar Phys. 98, 197-217.

Choudhuri, A.R. and Gilman, P.A. (1987), Astrophys. J. 316, 788-800.

Deinzer, W., Hensler, G., Schüssler, M. and Weishaar, E. (1984a), Astron. Astrophys. 139, 426-434.

Deinzer, W., Hensler, G., Schüssler, M. and Weishaar, E. (1984b), Astron. Astrophys. 139, 435-449.

Dungey, J.W. (1958), Cambridge University Press, Cambridge (IAU Symp. No. 6, ed. B. Lehnert) p. 135-140.

Duvall, T.L., Dziembcwski, W.A., Goode, P.R., Gough, D.O., Harvey, J.W. and Leibacher, J.W. (1984), Nature 310, 22-25.

Field, G.B. (1965), Astrophys. J. 142, 531-567.

Gaizauskas, V., Harvey, K.L., Harvey, J.W., and Zwaan, C. (1983), Astrophys. J. 265, 1056-1065.

Gilman, P.A. (1983), Astrophys. J. Suppl. 53, 243-268.

Gilman, P.A. and Miller, J. (1981), Astrophys. J. Suppl. 46, 211-238.

Glatzmaier, G.A. (1985), Astrophys. J. 291, 300-307.

Glencross, W.M. (1975), Astrophys. J. Letters 199, L53-L56.

Harvey, K. and Harvey, J. (1973), Solar Phys. 28, 61-76.

Hasan, S.S. (1985), Astron. Astrophys. 143, 39-45.

Krause, F. and Rädler, K.H. (1980), Pergamon Press, New York.

Machado, M.E., Moore, R.L., Hernandez, A.M., Revira, M.G., Hagyard, M.J., and Smith, J.B. (1988ג), Astrophys. J. 326, 425-450.

Machado, M.E., Xiao, Y.C., Wu, S.T., Prokakis, Th., and Dialetis, D. (1988b), Astrophys. J. 32€, 451-461.

Meyer, F., Schmidt, H.U., Weiss, N.O. and Wilson, P.R. (1974), Mon. Nat. Roy Astron. Soc. 169, 35-57. 
Moffatt, H.K. (1978), Cambridge University Press, Cambridge.

Parker, E.N. (1953), Astrophys. J. 117, 431-436.

Parker, E.N. (1955), Astrophys. J. 122, 293-314.

Parker, E.N. (1957a), Proc. Natl. Acad. Sci. 43, 8-14.

Parker, E.N. (1957b), Phys. Rev. 107, 830-836.

Parker, E.N. (1965), Astrophys. J. 142, 584-590.

Parker, E.N. (1966), Astrophys. J. 145, 811-833.

Parker, E.N. (1968), University of Chicago Press, Chicago. ed. B.M. Middlehurst and L.H. Aller.

Parker, E.N. (1969), Space Sci. Rev. 9, 651-712.

Parker, E.N. (1971a), Astrophys. J. 163, 255-278.

Parker, E.N. (1971b), Astrophys. J. 166, 295-300.

Parker, E.N. (1972), Astrophys. J. 174, 499-510.

Parker, E.N. (1979a), Cosmical Magnetic Fields, Oxford University Press, Oxford.

Parker, E.N. (1979b), Astrophys. J. 230, 905-913.

Parker, E.N. (1981), Astrophys. J. 244, 644-652.

Parker, E.N. (1982), Geophys. Astrophys. Fluid Dyn. 22, 195-218.

Parker, E.N. (1983a), Astrophys. J. 264, 642-647.

Parker, E.N. (1983b), Geophys. Astrophys. Fluid Dyn. 23, 85-102.

Parker, E.N. (1984), Astrophys. J. 283, 343-348.

Parker, E.N. (1987a), Solar Phys. 110, 11-21.

Parker, E.N. (1987b), Astrophys. J. 312, 868-879.

Parker, E.N. (1987c), Astrophys. J. 321, 984-1008.

Parker, E.N. (1987d), Astrophys. J. 321, 1009-1030.

Parker, E.N. (1987e), Solar Phys. 111, 297-308.

Parker, E.N. (1988), Astrophys. J. 330, 474-479.

Parker, E.N. (1989a), Geophys. Astrophys. Fluid Dyn. (in press).

Parker, E.N. (1990), Geophys. Astrophys. Fluid Dyn. (in press).

Rosner, R., Tucker, W.H. and Vaiana, G. (1978), Astrophys. J. 220, 643-665.

Ruzmaikin, A.A., Shukurov, A.M., and Sokoloff, D.D. (1988), Kluwer Academic Publishers, Dordrecht.

Soward, A.M. (1978), Astron. Nachr. 299, 25-33.

Spruit, H.C. (1974), Solar Phys. 34, 277-290.

Spruit, H.C. (1979), Solar Phys. 61, 363-378.

Stenflo, J.O. (1973), Solar Phys. 32, 41-63.

Stix, M. (1975), Astron. Astrophys. 42, 85-89.

Sweet, P.A. (1958), Cambridge University Press, Cambridge (IAU Symp. No. 6 ed. B. Lehnert) pp. 123-134.

Vrabec, D. (1971), D. Reidel Pub. Co., Dordrecht-Holland (IAU Symp. No. 43, ed. R. Howard). 
White, M.P. (1978), Astron. Nachr. 299, 209-216.

Zwaan, C. (1978), Solar Phys. 60, 213-240.

Zwaan, C. (1985), Solar Phys. 100, 397-414.

TRIMBLE: You mentioned the Maunder minimum period of reduced solar activity. What is known about epochs of unusually high solar activity?

PARKER: The so-called "Medieval Maximum" was the most recent century (the 12th) of hyperactivity of the Sun. It shows up clearly in ${ }^{14} \mathrm{C}$ production. One has no idea of what the sun was doing at that time. Presumably there were lots of sunspots, flares, plages, prominences, and an intense $X$-ray corona, compared to the "normal" activity maxima that we see today. The curious behaviour of terrestrial climate in step with the centuries of hyper- and hypo-activity on the sun is a controversial subject. Coincidence or a real connection?

KULSRUD: What is the nature of the discontinuity in the tangled field equilibrium?

PARKER: They are surfaces of discontinuity.

BENFORD: If coronal X-ray emission (including that near the loop footpoints) does not arise from wave dissipation, what mechanisms do you favor?

PARKER: I suggest that the principal energy source (heat source) of the solar $X$-ray corona is the dissipation at the tangential discontinuities (current sheets) that are an intrinsic part of the static equilibrium of almost all magnetic field topologies. That is to say, almost all continuous deformations of an initially uniform field result in the formation of internal tangential discontinuities as an intrinsic part of the equilibrium of the field.

The energy input to the coronal fields $\left(\sim 10^{2} \mathrm{G}\right)$ comes from the random motion $(\sim 0.5 \mathrm{~km} / \mathrm{s})$ of the footpoints of the bipolar fields in which the $\mathrm{X}$-ray emitting gas is confined. The necessary input of $10^{7} \mathrm{ergs} /$ $\mathrm{cm}^{2} \mathrm{sec}$ occurs when the field is wound up (on small scales) to the point that $\left\langle(\Delta \mathrm{B})^{2}\right\rangle / \mathrm{B}^{2} \simeq(1 / 4)^{2}$.

SOKOLOFF: There is a conservation law for knots number $\chi=\int \operatorname{HAd}^{3} \mathrm{x}$ in ideal MHD, but there are some possibilities to generate $\chi$ in non-ideal situations. So, the problem about $\chi$ is very important. What do you think about a possible progress in observational estimates of $\chi$ ?

PARKER: The topology of the magnetic field of a bipolar magnetic region is presumably without knots because the footpoints of each line of force are fixed in the photosphere. One could imagine that magnetic reconnection in a strongly deformed magnetic field might possibly create a true knot, but it is not obvious that this actually occurs. Consequently there is no application of knot theory to solar fields, so far as I am aware. 
DEINZER: Would you give some comments on the clustering of fibrils, Parker's spaghetti model? There seem to be observations on $p$-mode scattering on magnetic flux tubes recently.

PARKER: The clustering of fibrils to form sunspots is in opposition to the considerable magnetic pressure and can be forced only by a strong converging flow. There is no evidence at the surface for the necessary converging flow (originally proposed by Meyer, Schmidt, Weiss, and Wilson), but there is no other explanation possible, it seems.

The spaghetti model of the sunspot is suggested by the known fibril state of the surface fields that are swept together to form the spot. I was not aware of recent results from p-mode scattering, probing the subsurface structure of the sunspot.

FUnRT: You mentioned the flickering of the solar atmosphere in $\mathrm{X}$-rays. What is the amplitude of this flickering, and is there any report on a radioastronomical analogue?

PARKER: I am not aware of a radio detection of such flickering. The amplitude of the flickering is substantial, up to $50 \%$, on periods of 50-100 sec. There are several observational studies of transient luminosity and surface brightness variations. The references are listed and summarized in a paper on nanoflares and the solar $X$-ray corona (1988, ApJ 330, 47).

DOLGINOV: How is the poloidal magnetic field restoring in the overshoot region where the velocities are very low? Have you got any quantitative estimations?

PARKER: I find it difficult to understand a dynamo operating in a thin overshoot layer below the convective zone, for exactly the reasons that you mention. The large quantities of flux erupting through the surface of the Sun over a period of many months suggests that the azimuthal field must be at least $3.10^{3} \mathrm{G}$ over a depth of the order of $10^{5} \mathrm{~km}$, or stronger over a smaller depth. Overshoot dynamos are usually assumed to occupy a thin layer $\left(\sim 10^{4} \mathrm{~km}\right)$, requiring $B_{\phi} \geqslant 10^{4} \mathrm{G}$. I do not understand how so strong a field can be manipulated by weak convection $(\leqslant 10 \mathrm{~m} / \mathrm{s})$ to produce a dynamo.

My guess is that the dynamo operates in the lower half of the convective zone.

RUZMAIKIN: Do you consider as realistic the absence of a radial gradient of angular velocity in the solar convective zone?

PARKER: On the one hand, helioseismology indicates only a small gradient in $\Omega$ in the solar convective zone at low latitude, with $\Omega$ decreasing slowly inward. On the other hand, numerical simulations provide cases where $\partial \Omega / \partial r>0$ and $\partial \Omega / \partial r<0$, depending upon how the numerical model is set up (with or without density stratification, lower boundary conditions, etc.). Hence I have no basis for disbelieving the result inferred from helioseismology. 
MOUSCHOVIAS: Granted that we have learned a lot about astrophysical magnetic fields from the Sun, but wouldn't you agree that extrapolation of that knowledge to the interstellar medium (especially in cloud interiors), where the magnetic pressure usually dominates the thermal (and turbulent) pressure, would be very dangerous?

PARKER: Any extrapolation is purely conjectural and must be established by observation before it can be taken seriously. I should emphasize that point. I describe the solar-galactic similarities here only so that observers will be aware of the possibilities.

As a matter of fact, the turbulent kinetic energy density $1 / 2 \rho \mathrm{v}^{2}$ of the interstellar medium is not small compared to $B^{2} / 8 \pi$. Similarly the cosmic-ray pressure and energy density are not small compared to $\mathrm{B}^{2} / 8 \pi$. So the interstellar field is strongly deformed by the matter, providing the theoretical possibilities for magnetic activity that I have described. 